\title{
„Es muss nicht immer Case Management drauf stehen, wenn es Case Management ist"
}

Interview mit Sabine Peer

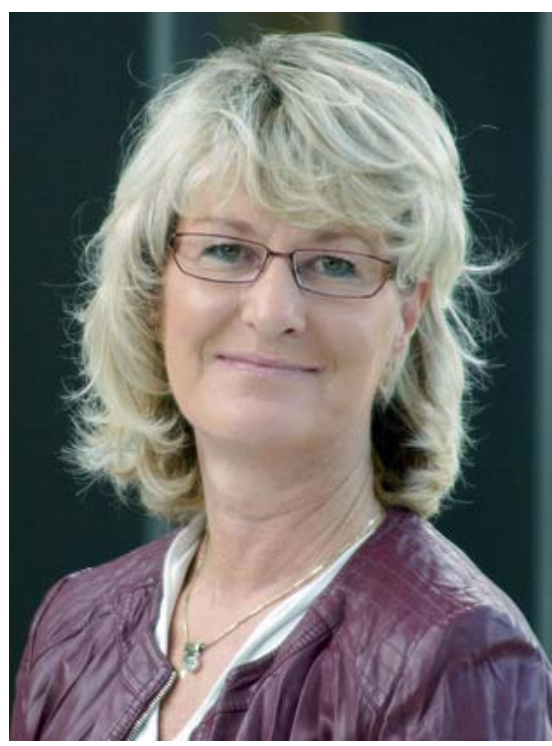

Die Leiterin des Case Managements eines Klinikums der Maximalversorgung erklärt die praktische Arbeit einer guten Nachsorge für die Patienten. Und argumentiert, warum sich der Aufwand nicht nur für den Patienten, sondern auch für ein Krankenhaus rechnet.

Wie viele Patienten betreuen Sie mit Ihrem Team?

Etwa 7000 Fälle pro Jahr. Wir haben rund 900 Planbetten in 28 Kliniken und Instituten hier am Hauptstandort in Lüdenscheid. Hinzu kommen noch 2 externe Standorte, das Marienhospital Letmathe mit 90 Betten und die Stadtklinik Werdohl mit 110 Betten. Den Standort Werdohl, an dem unsere akutgeriatrische Station ist, betreuen wir mit. Ich habe in meinem Team 11 Mitarbeiter auf genau 8,54 Vollzeitstellen. Darunter sind Pflegekräfte, aber auch Sozialarbeiter oder Sozialpädagogen. Wir bilden eine eigene Abteilung, sind aber der Pflege zugeordnet [1].

Und mit 8 Vollzeitstellen bieten Sie ständig an die 1000 Patienten Case Management?

Nein, so dürfen Sie nicht rechnen, wir betreuen ja immer nur einen Teil der Patien- ten. Im Schnitt sind es pro Jahr an die 6500 bis 7000. Eine Vollkraft schafft ca. 25 Fälle proTag. Und im Durchschnitt arbeitet eine Vollkraft für jeden betreuten Patienten ca. 180 Minuten während seines gesamten stationären Aufenthalts. Aktuell können wir uns so gleichzeitig immer um etwa 200 Patienten kümmern.

Und die Patienten in den gut 800 andere Betten kriegen keinen Case Manager?

So ist es, wir kümmern uns um die, die unseren Service brauchen. Es wird nicht jeder Patient einen Case Manager sehen.

\section{Und wer sieht ihn?}

Das hängt weniger von der Indikation ab, sondern von Faktoren im Hintergrund. Wir filtern die Patienten bereits bei der Aufnahme. Die Filterkriterien sind in Anlehnung an ein geriatrisches Assessment konzipiert. Ein Kriterium ist das Alter, ein anderes, wie oft jemand schon im Krankenhaus war, ob das in kürzeren Abständen hintereinander war, es kommt darauf an, ob man Einschränkungen in den Aktivitäten des täglichen Lebens hat, den ATLs. Eine chronische Wunde, das Vorliegen einer Pflegestufe, das sind weitere Faktoren, die die Chancen auf Case Management steigen lassen, denn solche Fälle haben in der Regel einen höheren Versorgungsbedarf und sie, ich sage mal, kippen oft nach der Entlassung. Da steuern wir gegen.

\section{Befragen Sie die Patienten dafür?}

Nein, die Ärzte oder die Schwestern melden uns die Ergebnisse des Screenings, das schon bei der Aufnahme stattfindet, über das KIS (Krankenhausinformationssystem). Innerhalb von 24 Stunden nach der Aufnahme ist dann einer unserer Case Manager auf der Station und schaut sich den Fall an, prüft in Zusammenarbeit mit der Pflege, wo wirklich Bedarf bestehen könnte. Die Pflegekräfte sind meist am nächsten an den Fall dran. Bei ihnen holen wir uns die Auskunft, was der Patient hat, wie er gerade versorgt wird, was für Einschränkungen er mitbringt und was es im sozialen Umfeld gibt. Entscheiden wir ja das ist jemand, dem wir Case Management anbieten wollen, gibt es mit dem Arzt eine enge Abstimmung, wann die Entlassung sein soll, und dann nehmen wir direkt mit dem Patienten Kontakt auf.

Sie kommen zum Patienten erst, wenn er entlassen wird?

Nein. Direkt, möglichst früh schon nach der Aufnahme.

Was bieten Sie an? Denken wir an einen älteren Patienten mit einem Oberschenkelbruch.

Das ist sicher eine Zielgruppe, denn solche Patienten müssen unbedingt rasch wieder mobilisiert werden. Ich gucke dann erstmal, ob es ein soziales Umfeld gibt, einen Lebenspartner, Kinder. In welcher Wohnsituation lebt der Patient? Vielleicht schon im Heim, im betreuten Wohnen, hat er eine Pflegestufe? Wie mobil ist er? Unser Ziel ist es, alles zu tun, dass die Patienten auch nach der Entlassung wieder in ihr gewohntes Umfeld zurückkehren können.

\section{ZUR PERSON}

Die diplomierte Krankenpflegerin Sabine Peer (Jahrgang 1965) hat seit 2005 am Klinikum Lüdenscheid (Kommunaler Krankenhausverbund Märkische Kliniken $\mathrm{GmbH}$ ) eine Abteilung für Case Management neu aufgebaut. Die gebürtige Sauerländerin absolvierte zuvor einen Masterstudiengang in Gesundheitswissenschaften und sammelte diverse Weiterbildungen, darunter auch die zum Case Management nach den Richtlinien der Fachgesellschaft DGCC. Beim Start ihrer neuen Abteilung im April 2005 bekam Peer ein Büro mit Telefon, heute arbeiten in ihrem Team 11 Leute auf gut 8 Vollzeitstellen.

E-Mail: sabine.peer@

klinikum-luedenscheid.de 


\section{Und was tun Sie dafür?}

Wir diskutieren zum Beispiel oft, in wie weit eine Anschlussheilbehandlung möglich und sinnvoll wäre. Wir haben sehr gute Erfahrungen damit gemacht, wenn die Patienten nach der Akutbehandlung noch für 2 bis 3 Wochen in unsere geriatrische Station gehen, wo sie medizinisch-pflegerisch gut versorgt werden.

\section{Wer zahlt das?}

Die Kasse, das geht über die Direktverlegung in die geriatrische Station und läuft als sogenannte Komplexbehandlung.

Die Entscheidung über Anschlussheilbehandlung in der Geriatrie werden am Ende aber die Ärzte im Krankenhaus treffen?

Ob ein Patient in der Geriatrie angemeldet wird, steuern und entscheiden wir vom Case Management. Die Aufnahmeentscheidung in der Geriatrie trifft die dortige Chefärztin. Aber es wird kein Patient in der Geriatrie angemeldet, ohne dass ein Mitarbeiter vom Case Management zuvor drauf geguckt hat. Sonst ginge das oft schief.

\section{Wieso?}

Wir können und möchten auch dafür sorgen, dass keine Patienten in die Geriatrie verlegt werden, von denen wir annehmen müssen, dass sie nicht davon profitieren werden.

\section{Zum Beispiel?}

Patienten, die schon im Heim leben, die schon immobil waren, bevor sie den Knochenbruch erlitten, die eine Pflegestufe 2 oder 3 haben oder die vielleicht schon dement sind - all das sind Ausschlusskriterien für einen Aufenthalt in der Geriatrie. Diese Form der Behandlung ist dann nicht zielführend.

Die Entscheidung gegen eine Verlegung in die geriatrische Station bedeutet für solche Patienten aber erst recht noch lange keine Hilfe.

Ja, und unsere Aufgabe ist es dann, auf anderem Weg dafür zu sorgen, dass die Brüche am Übergang zurück aus dem Krankenhaus bestmöglich überbrückt werden. Wir haben zum Beispiel in unserem Haus ein PIZ (Patienteninformationszentrum) für Angehörige, die familiäre Pflege leisten. Das ist ein Modellprojekt seit 2001, mit der Uni Bielefeld, mit verschiedenen AOKs, an dem alle Kassenpatienten kostenlos teilnehmen können und was wir dann Angehörigen vermitteln, damit sie gut vorbereitet werden.

Die Mitarbeiter aus dem PIZ können im Rahmen der Angehörigenschulung die Patienten auch bis zu 6 Wochen nach ihrer Entlassung im häuslichen Bereich aufsuchen, auch mehrfach, um dort zu helfen und zu beraten. Das Stichwort ist hier die aufsuchende Pflege.

Darüber reicht der Arm des Krankenhauses quasi auch bis zur nachfolgenden ambulanten Versorgung?

Genau. Ganz grundsätzlich gilt, sobald wir rückgespiegelt bekommen, dass etwas hakt oder wenn vorzeitige Wiederaufnahmen da sind, dann kommen wir ins Spiel. Denn es geht uns um eine generell gute Versorgungsqualität und zugleich auch um Kostensenkung. Das Krankenhaus möchte durch gutes Case Management auch vermeiden, dass die Patienten innerhalb einer bestimmten Zeit wieder ins Klinikum kommen.

\section{Gelingt das denn?}

Ja, das gelingt. Dafür haben wir vor 3 Jahren die Akten zu rund 6000 Patienten nachbearbeitet - und bei den Patienten, die wir im Case Management hatten, fanden sich gerade mal 6 Wiederaufnahmen, die im Übrigen alle medizinisch begründet waren. Das waren keine Versorgungsprobleme.

Und haben Sie dagegen auch eine Ziffer, was wäre, wenn kein Case Management stattfindet?

Leider nicht, denn man kann da keine Studie drüber machen, das ist ethisch nicht vertretbar. Was wir wissen, ist, dass wir die durchschnittliche Verweildauer durch Case Management verkürzen konnten, wir kommen im Durschnitt auf 2 bis 4 Tage, die wir pro Fall einsparen. Wir haben hier am Klinikum Lüdenscheid heute eine durchschnittliche Verweildauer von 5,89 Tagen.

Auf diese Zahlen kommen Sie durch einen Vorher-Nachher-Vergleich, vor und nach Einführung von Case Management? Richtig. Details dazu kann ich nicht sagen. Aber ich kann sagen, dass wir uns als Abteilung für das Krankenhaus rechnen - eine gute Koordination der Versorgung kostet ein Krankenhaus etwas, am Ende aber spart es durch vermiedene Wiederaufnahmen und eine oft verkürzte Verweildauer.

Wie viele Krankenhäuser haben so eine Abteilung wie Ihre, in Deutschland?

Nach meiner Schätzung an die $40 \%$. Es ist leider immer noch so, dass $60 \%$ der Häuser weder ein Entlassungsmanagement noch ein Case Management noch sonst irgendwas haben. Die machen das auf Zuruf, was eigentlich nicht funktioniert.

Auch gibt es unterschiedliche Auslegungen. Es gibt Häuser, die haben ein Case Management in der Verwaltung angesiedelt, beim Controlling, da liegt der Schwerpunkt auf der Codierung. Das hat aber mit unserer Arbeit nichts zu tun. Auch Dinge wie das Belegungsmanagement packen manche Häuser unter Case Management, aber auch das liegt bei uns in Händen der Stationsleitung. Auch die Terminierung der Großgeräte oder der OPs. Das ist in meinen Augen alles kein Case Management. Der Begriff Case Management ist ungeschützt, es kann sich jeder so einen Zettel ans Büro kleben.

Angenommen, Sie hätten einen Patienten nach einer schweren Amputation. Inwieweit helfen Sie da in der Nachsorge? Sind die Voraussetzungen gegeben, beantragen wir eine Anschlussrehabilitation. Wir begleiten die Anpassung einer Prothesenversorgung. Wir beraten zum Thema Schwerbehinderung, kümmern uns bei Bedarf auch um den Antrag auf einen Schwerbehindertenausweis. Wir schalten teilweise das Patienteninformationszentrum mit ein.

Übergeben Sie am Ende an einen Case Manager, den ein ambulanter Pflegedienst hat, sobald der übernimmt?

Es ist gut, wenn Dienste so eine Fachkraft haben. Bei Amputationen wäre ambulante Pflege in der Regel aber nicht besonders sinnvoll. Wir haben da andere Netzwerke, die mit eingebunden werden. Je nach Bedarf helfen wir mit Anträgen und Informationen zur hauswirtschaftlichen Tätigkeit, Begleitung zum Arzt, Menüservice.

Bei anderen Patienten kümmern wir uns, falls erforderlich, natürlich mit um die Beantragung von Leistungen der Pflege, um 
die Beauftragung eines ambulanten Pflegediensts und benennen Kontaktpersonen, außerhalb der Klinik, an die sich ein Patient bei Fragen wenden kann.

Kann es sein, dass Sie jemanden noch Wochen nach der Entlassung an der Strippe haben?

Sobald jemand einen Pflegedienst hat, greifen wir normalerweise nicht mehr ein. Wohl aber sagen wir den Patienten und Angehörigen, wenn Sie mal völlig ratlos sind, dann rufen Sie uns an. Wir versuchen auch dann, den richtigen Ansprechpartner zu finden.

Suchen Sie auch eine Rehaklinik bei Bedarf aus?

Wir können informieren, die Entscheidung liegt beim Patienten. Wir helfen aber bei den Formalitäten. Es gibt ein paar Spielregeln, viele Patienten wissen nicht, dass sie die Freiheit haben, sich das Haus selbst auszusuchen.

$\mathrm{Na}$, dann wollen jetzt aber alle nach Sylt? (Lacht), geht nicht, dort gibt es keine Rehaklinik. Auch sollte das Haus in einem Aktionsradius von $200 \mathrm{~km}$ um den Wohnort sein, innerhalb des Bundeslands. Der Patient hat Wunsch und Wahlrecht nach $\S 9$ SGBIX und $\S 33$ SGBI. Wir unterstützen die Patienten bei der Auswahl der geeigneten Rehaeinrichtung und auch beim Widerspruchsverfahren.

\section{Noch ein paar Tipps?}

Mit dem Krankenhausstrukturgesetz sind 20152 Maßnahmen in Kraft getreten, um Menschen, die eine Versorgungslücke haben, die Möglichkeit zu geben, auch ohne eine Pflegestufe entsprechende Leistungen zu bekommen. Das wäre einmal der Antrag auf häusliche Krankenpflege ohne Pflegebedürftigkeit. Das geht nach §37a SGB V Absatz 1. Voraussetzung für die Bewilligung von der Kasse ist, dass der Patient deutliche Einschränkungen in seiner Mobilität hat, sich nicht selber helfen kann, kein soziales Netzwerk hat, also vor allem keinen Lebenspartner, der daheim helfen könnte.

Wenn ich sehe, der Patient bekommt keine Pflegestufe, dann versuche ich das über diese Schiene.
Für wie lange lässt sich das dann beantragen?

Wir können laut Krankenhausstrukturgesetz für längstens 7 Tage eine Verordnung mitgeben über diese Grundpflege. Ich beantrage das hier im Krankenhaus ab dem Datum der Entlassung und schreibe auf den Antrag bis auf Weiteres. Eine evtl. nötige Verlängerung ist Sache des nachsorgenden Arztes.

Und die zweite Möglichkeit für Pflege auch ohne Pflegestufe?

Ist eine stationäre Kurzzeitpflege nach $\S 39 c$ SGB V. Wenn dieser Antrag bewilligt wird, zahlt die Krankenkasse den Satz von 1612 Euro dazu, für eine 4-wöchige Kurzzeitpflege.

Was, wenn ich solche Information und Hilfe über ein Case Management erst gar nicht kriege? Prinzipiell habe ich als Versicherter nach SGB V einen Anspruch auf Versorgungsmanagement wie auf Entlassungsmanagement im Krankenhaus.
Was tun, wenn man mir das alles gar nicht bietet?

Ansprechpartner für Beschwerden wäre primär die jeweilige Kasse. Der Punkt ist, dass es im Gesetz bei Nichterfüllung keine Regeln für Sanktionen gibt. Da sind die Formulierungen meistens ein bisschen schwammig.

Es muss andererseits auch nicht unbedingt immer Case Management draufstehen, damit man Case Management bekommt. Es kann in einem anderen Krankenhaus vielleicht auch Patientenmanagement heißen, Überleitung, Entlassmanagement, es gibt unterschiedliche Begrifflichkeiten. Zumindest Teile von Case Management können solche Einrichtungen schon bieten. Aber nicht alles. Pflegeüberleitung kann sich vorbildlich um die Anträge auf häusliche Pflege kümmern. Doch Case Management macht mehr. Wir betreuen hier Patienten aller Altersstufen, von 0 bis über 100 Jahre. Es gibt leider auch schon schwer kranke Kinder. Wir organisieren palliative Begleitung - für alle Altersgruppen. Unter Umständen bis ins Ausland. 


\section{Wie das?}

Wir haben einen speziellen Fall gehabt, da wollte eine Dame, die aus Anatolien kam, und deren Prognose schlecht war, zu Hause sterben. Unterstützt durch einen Förderverein wurde ein Flug nach Anatolien organisiert. Zuvor wurde versucht, einen Arzt vor Ort ausfindig zu machen. Das Case Management organisiert so was nicht alleine. Aber wir wirken daran mit, dass Patienten solche Wünsche möglichst erfüllt werden können.

\section{Aber einmal im Jahr müssen Sie trotzdem der Chefetage Zahlen vorlegen, dass sich Ihre Abteilung rechnet?}

Ja, und wir gehen deshalb auch aus Kostengründen in manchem Einzelfall auch schon mal tiefer in die Diskussion mit dem Arzt. Es kommt vor, dass wir zu einer früheren Entlassung raten können und das dann auch tun, weil die Anschlussbehandlung schon organisiert werden konnte. Aber das Wohl des Patienten muss immer im Mittelpunkt stehen. Es nützt auch ökonomisch gar nichts, wenn ich kurzfristig wirt- schaftlich denke. Dann wird das ein Bumerang, dann kommt der Patient zurück.

Andererseits müssen auch hinter einem Drehtüreffekt nicht immer medizinische Gründe stecken.

\section{Sondern?}

Denken Sie in Richtung Verwahrlosung, Alkoholiker, Missbrauch. Die Palette ist breit gestreut.

Wenn also in der Aufnahmeakte steht trinkt viel Alkohol, dann stehen Sie am nächsten Tag am Bett?

Nicht unbedingt. Angenommen, Sie können sich noch alleine waschen, alleine wohnen, ohne dass anderen Personen irgendwas auffällt, dann unternehmen wir zunächst mal nichts. Jeder hat ein Recht auf Verwahrlosung.

\section{Ein kerniger Satz.}

Das ist aber so, wenn ein Patient keine Hilfe möchte. Andererseits kann es nicht sein, dass ein Alkoholiker jede Woche hier mit einer neuen Platzwunde oder Fraktu- ren aufläuft, die Wohnung ist womöglich schon verwahrlost - dann ist das doch ein deutlicher Hinweis darauf, dass jemand nicht mehr in der Lage ist, für seinen Gesundheitszustand selbst Sorge zu tragen. Dann sollten wir gemeinsam über die Einrichtung einer Betreuung oder vielleicht über ein ambulant betreutes Wohnen nachdenken.

Aber klar, ohne den Patienten und seine Zustimmung geht auch das alles nicht. Den Datenschutz schreiben wir ganz groß.

\section{Literatur}

[1] Vorstellung des Case Managements im Klinikum Lüdenscheid beim Projekt „Wegweisende Modelle zur Weiterentwicklung der Pflege im Krankenhaus" des Bundesministeriums für GesundheitS_KE;). Im Internet: http://www.pflege-krankenhaus.de/neuearbeitsteilung/modelle-im-ueberblick/ klinikum-luedenscheid-einfuehrung-voncasemanagement/daten-zum-modell.html; Stand: 26.01.2017

Das Interview führte Bernhard Epping 